\title{
It does matter who we are grateful to: A latent profile analysis
}

\section{Edyta Charzyńska ${ }^{1}$ (D)}

Published online: 18 July 2019

(C) The Author(s) 2019

\begin{abstract}
Scientific interest in trait gratitude has burgeoned in recent years. However, the majority of studies on this subject do not take into account that the level of gratitude may differ depending on the target. The primary aim of this study was to examine the heterogeneity of a sample of 765 young adults with respect to gratitude toward different interpersonal targets. Six targets of gratitude were considered: mother, father, grandparent(s), sibling(s), partner, and friend(s). Using latent profile analysis, five profiles of young adults with similar combinations of gratitude toward different people were identified: (1) primarily grateful to parents $(35.4 \%)$, (2) primarily grateful to mother and partner (24.1\%), (3) highly grateful to all measured targets (17.1\%), (4) primarily grateful to partner and friend(s) (13\%), and (5) not highly grateful to any measured target $(10.4 \%)$. Latent profiles differed in terms of gender, well-being and coping strategies. The study suggests that considering different interpersonal targets of gratitude and using the person-oriented approach may considerably deepen the knowledge on trait gratitude and its relationship with other variables.
\end{abstract}

Keywords Gratitude $\cdot$ Person-oriented approach $\cdot$ Latent profile analysis $\cdot$ Well-being $\cdot$ Coping strategies

\section{Introduction}

Within the last 20 years, there has been an increasing interest in psychology in investigating traits that both help a person live a good and happy life and contribute to the well-being of others (Peterson and Seligman 2004). One of these traits (called character strengths in positive psychology) is gratitude, defined as a generalized tendency (life orientation) at perceiving and appreciating life's positive aspects (Wood et al. 2010).

Many studies have confirmed the beneficial role of gratitude in human health. A grateful disposition is related to higher well-being, satisfaction with life, hope and lower stress, anxiety and depression (McCullough et al. 2002; Wood et al. 2010; Wood et al. 2008). People with higher gratitude evaluate their physical health as better, report fewer physical symptoms, and more often exercise (Emmons and McCullough 2003; Hill et al. 2013). Moreover, gratitude is beneficial for social functioning: it facilitates establishing and maintaining relationships, strengthens interpersonal relationships, and

Electronic supplementary material The online version of this article (https://doi.org/10.1007/s12144-019-00361-3) contains supplementary material, which is available to authorized users.

Edyta Charzyńska

edyta.charzynska@us.edu.pl

1 University of Silesia, ul. Grażyńskiego 53, 40-126 Katowice, Poland stimulates cooperation and prosocial attitudes (Algoe et al. 2008; Ma et al. 2017).

The majority of previous studies on trait gratitude have focused on exploring gratitude as a unidimensional construct or investigating the within-person characteristics of gratitude such as its facets, elements, components, or forms (Hammer and Brenner 2019; Lin 2017; McCullough et al. 2002; Morgan et al. 2017; Watkins et al. 2003). Despite the merits of those studies, they also have a serious limitation: they usually did not consider context-specific characteristics of trait gratitude. Having said that, the contextualization of gratitude research is important as gratitude-related emotions, thoughts and behaviors occur in a specific context; as such, they may not be sufficiently captured with general constructs (Chen 2017).

The current study is focused on one of the contextual characteristics of gratitude: its explicit targets. It relies on the assumption that gratitude can target different people to different degrees. This assumption is analogous to the one adopted over a decade ago in studies of forgiveness, which is often viewed as a character strength closely related to gratitude (Luthans et al. 2015). For many years, forgiveness had been associated with forgiving others (Enright and The Human Development Study Group 1991). Currently, however, many studies take into account that forgiveness may be oriented toward different targets (e.g., oneself, others, God; Toussaint and Webb 2005). Importantly, studies showed that a) people may display different levels of forgiveness depending on its target (Toussaint 
et al. 2001; Webb et al. 2006), b) different targets of forgiveness may exert a different effect on functioning of the individual who is doing the forgiving (Macaskill 2012; Svalina and Webb 2012), and c) distinct factors determine the levels of forgiveness toward different targets (Hall and Fincham 2005; Ross et al. 2004).

The results of studies on forgiveness lead to the assumption that in the case of gratitude as well, investigating contextspecific factors such as the targets of gratitude may be fruitful, providing in-depth understanding of the complex nature of this character strength. The results of several studies concerning specific targets of gratitude provide additional arguments for these assertions. For example, using daily diary methodology, Gordon et al. (2011) noted that among long-term married couples, feeling grateful to spouse was significantly related to one's own marital satisfaction and predicted the spouse's satisfaction as well (see also: Algoe et al. 2008). In another field of research, Rosmarin et al. (2011) showed that gratitude toward God mediated the relationship between trait gratitude and religious commitment. This study also revealed that the interplay between religious commitment and gratitude toward God added unique variance in predicting mental well-being, over and above general gratitude. In a recent nationwide survey, Krause et al. (2017) verified relationships between general feelings of gratitude, gratitude to God, and an indicator of blood sugar control (hemoglobin A1c; HbA1c). General gratitude was associated with lower levels of $\mathrm{HbA} 1 \mathrm{c}$ regardless of gender. By contrast, a negative relationship between gratitude toward God and HbA1c occurred only for women. A contextualized understanding of gratitude was also introduced to sports research. For instance, the study conducted by Chen and Chang (2017) showed that sports-domain gratitude (comprising three targets of gratitude: one's coaches, teammates and sport-related experiences) explained the variance in athletes' burnout and team satisfaction, above and beyond domaingeneral gratitude.

One limitation of studies on targets of gratitude is that most of them have been focused on merely one or two specific targets, without taking into account the associations among the levels of gratitude toward multiple interpersonal targets. This study aims to fill this gap, deepening the knowledge about targets of gratitude toward different people and pushing forward the research on the relationship between this contextual characteristics of gratitude and other outcomes such as well-being or coping strategies.

Another limitation of studies on targets of gratitude is that they employ the variable-oriented approach, which assumes that people in a particular sample are drawn from a single homogeneous population, and thus relationships between variables are treated as identical across all individuals (Bergman et al. 2003; Collins and Lanza 2010). The person-oriented approach overcomes these limitations. It allows one to study the similarities and differences among individuals with consideration to how variables relate to each other. In this approach, it is assumed that the population is heterogeneous, and the goal of the analysis is to distinguish subpopulations that group people with similar traits (i.e., groups that are internally homogeneous; Berlin et al. 2014). It also assumes that population subgroups differ meaningfully in various characteristics and relationships (Bergman et al. 2003). With consideration to these reasons, the person-oriented approach was chosen as an optimal technique for capturing potentially elaborate patterns of trait gratitude toward different people.

\section{Current Study}

The primary purpose of this study was to identify distinct profiles of individuals with similar combinations of levels of gratitude toward different interpersonal targets. The following targets of gratitude were taken into account: gratitude toward one's mother, father, grandparent(s), sibling(s), partner, and friend(s). The targets of gratitude were chosen with respect to the phase of development of the participants (i.e., young adulthood) and the developmental tasks they faced (Glover 2000; Lenz 2001). Hence, the study involved both members of the family of origin and non-family close people.

To the best of the author's knowledge, no previous studies could serve as a direct source of hypothesis on the number and shape of profiles of gratitude levels toward different people. Therefore, this research is exploratory in nature, and so no specific hypotheses on the exact number and shape of profiles were formulated. Nonetheless, it was assumed that there would be at least three distinct profiles: one grouping people with high levels of gratitude toward all measured interpersonal targets, one with low levels of all these variables, and at least one with diversified levels of gratitude depending on its target.

The secondary aim of this study was to explore the relationships between the levels of gratitude toward different targets, covariates, and outcome variables. More specifically, gender was examined as a potential predictor of latent profile membership. Because some evidence (e.g., Froh et al. 2010; Kashdan et al. 2009) suggests that women may have higher trait gratitude than men, it was expected that the proportion of women to men will be higher in the profiles with higher levels of trait gratitude toward different targets than in profiles with lower levels of these variables. Moreover, the study investigated whether people who follow distinct patterns of gratitude toward interpersonal targets differ significantly in terms of well-being and coping strategies. Several experimental and correlational studies have confirmed a positive relationship between gratitude and well-being (McCullough et al. 2002; Nezlek et al. 2019; Wood et al. 2010). As for the relationship between gratitude and coping strategies, the evidence is rather scarce. Nevertheless, some studies have suggested that for grateful people, coping strategies may serve as 
psychological resources. For instance, Wood et al. (2007) found that grateful people more often used adaptive coping (namely, emotional and instrumental support, active coping, positive reinterpretation, and planning) and less frequently engaged in maladaptive coping (namely, behavioral disengagement, substance use, self-blame, and denial) than people with low gratitude. Lau and Cheng (2017) found similar results in a study carried out among 101 Chinese familial caregivers of people with dementia. This study found that gratitude was positively related to positive reframing, acceptance, humor, emotional social support seeking, and religious coping. Given these results, in the current study, people who belong to the profile with high levels of trait gratitude toward all measured interpersonal targets are predicted to display higher well-being and use more constructive coping strategies than members of the profile with low levels of gratitude regardless of the target. Due to the lack of relevant theoretical premises, no specific hypotheses regarding members of profile(s) with diversified levels of gratitude depending on its target were formulated. Nevertheless, it was expected that those people will significantly differ in well-being and coping strategies in contrast to the two more uniform profiles.

\section{Methods}

\section{Participants}

The study was carried out among 1,183 students of five universities in the southern part of Poland. Only data obtained from people who had sibling(s) and were in a romantic relationship were used in the current analyses. Thus, calculations were performed for 765 students, including 488 women $(63.8 \%)$ and 277 men $(36.2 \%)$. The mean age of the participants was $20.53(S D=2.33)$. Most of them were students of social sciences $(66.4 \%)$, in their first year $(85.3 \%)$, and studying full-time (82.1\%). Almost two-thirds of the students lived with their parents, $21.8 \%$ rented flats, $7.1 \%$ had their own flats, and $5.1 \%$ lived in dormitories.

\section{Measures}

\section{Gratitude toward Different Interpersonal Targets}

Levels of gratitude toward different people were measured with single items, beginning with the phrase: "How grateful are you to your...?" The following targets of gratitude were included: mother, father, grandparent(s), sibling(s), partner, and friend(s). Responses were collected using a unipolar 5point scale with only the endpoints labeled $(1=$ "I am not grateful at all." and $5=$ "I am very grateful."). The measure is presented in Online Resource 1.
Single items for measuring gratitude toward different interpersonal targets were chosen in this study for several reasons. To begin with, the author intended to reduce fatigue and frustration in the respondents that could result from answering a high number of similar questions. This was done after consideration of research results showing that survey fatigue increases response bias and reduces data completion and data quality (Fan and Yan 2010; Meade and Craig 2012; Rolstad et al. 2011). Moreover, using single items to measure psychological constructs is recommended when the construct is narrow in scope, concrete, unidimensional, and unambiguous to the respondent (Rossiter 2002; Sackett and Larson 1990). According to Kashdan et al. (2009), gratitude is a simple construct for people to grasp; thus, it can be measured successfully with one or two items. This assumption has been also confirmed by the results of studies on gratitude in which very short measures were used instead of multiple-item scales (Graham 1988; Jans-Beken et al. 2018; Layous et al. 2017; Visserman et al. 2018). Taking into consideration these premises and the fact that in this study the respondents were asked about levels of gratitude toward specific, well-defined persons from their lives, measurement using single items was found to be adequate and sufficient.

So as to ensure the quality of data collected using the measures of gratitude, the test-retest reliability method for gratitude items was applied. A subsample of the participants (384 students: 231 women and 153 men) completed this measure again 6 weeks after the first assessment. Test-retest reliability coefficients ranged from .74 for gratitude toward the partner to .85 for gratitude toward the mother, indicating satisfactory reliability of the applied measures.

\section{Well-Being}

Mental Health Continuum-Short Form (MHC-SF; Keyes 2002) in the Polish adaptation by Karaś et al. (2014) was used to measure the participants' well-being. MHC-SF is comprised of 14 items constituting three facets of well-being: emotional (3 items), social (5 items) and psychological well-being (6 items). The participants answered using the following response options: "never," "once or twice," "about once a week," "about two or three times a week," "almost every day," and "every day." The indices of particular facets of well-being were obtained by adding the responses to particular items. In this study, Cronbach's alpha coefficient for the three subscales of MHC-SF ranges from .84 ("emotional well-being") to .87 ("psychological well-being").

\section{Coping Strategies}

Coping strategies were measured with the Polish adaptation (Juczyński and Ogińska-Bulik 2009) of 28-item Brief COPE (Carver 1997), which is an abridged version of the 60-item 
COPE Inventory (Carver et al. 1989). Brief COPE includes 14 two-item subscales: 1) self-distraction, 2) active coping, 3) denial, 4) substance use, 5) use of emotional support, 6) use of instrumental support, 7) behavioral disengagement, 8) venting, 9) positive reframing, 10) planning, 11) humor, 12) acceptance, 13) religion, and 14) self-blame. In this study, Brief COPE was adjusted to measure situational coping, namely, coping with stressful academic situations. The participants were asked to assess whether and to what extent they used particular coping strategies when facing stressful situations in the university setting. Several examples of academic stressful situations were given to the participants, i.e., taking a test or an exam, giving an oral presentation to the class, having an argument with a professor or another student, having too much academic work to do, and feeling lonely or isolated. The respondents evaluated each item on a 4-point rating scale ranging from 0 to $3(0=$ "I haven't been doing this at all," $1=$ "I've been doing this a little bit," 2 = "I've been doing this to a medium extent," 3 = "I've been doing this a lot"). Subscale scores were derived by adding the responses to relevant items. In this study, internal consistency assessed using Cronbach's alpha coefficient was satisfactory for all coping strategies (from $\alpha=.62$ for "venting" to $\alpha=.90$ for "substance use" and "religion").

\section{Procedure}

All respondents were informed about the anonymity and confidentiality of the study, voluntary participation, and their right to withdraw from the study at any point. Each participant was given the research questionnaire and was asked to fill it out within two weeks. To minimize the number of refusals to participate, when handing out the set of questionnaires, the researcher emphasized that the questionnaires were short and completing them would not take more than 10 to $15 \mathrm{~min}$ (Galesic and Bosnjak 2009). In addition, when handing out the questionnaires, the researcher emphasized it was important to complete the questionnaires carefully and diligently and asked the respondents to check that all questions were answered before returning the questionnaires. The same request was also written on the last page of the questionnaires. Apart from that, questionnaire design and layout were planned so as to reduce boredom or disengagement from the research (Toepoel et al. 2009). Thus, a maximum of 14 items were presented on each page. The questionnaires were given back by $92 \%$ students who were asked to participate.

\section{Data Analysis}

The analyses were preceded by exploration of missing data mechanisms and patterns of missingness. Little's Missing Completely at Random (MCAR) test (Little and Rubin 1987) was applied to examine whether data were missing completely at random. Missing data were imputed with maximum likelihood (ML) estimates using the expectationmaximization (EM) algorithm (Allison 2003). After imputation, descriptive statistics, along with correlation coefficients for different interpersonal targets of trait gratitude, were calculated. Then, means for gratitude toward different people were compared with each other using repeated ANOVA contrasts.

Next, to identify latent profiles with distinct patterns of gratitude toward different targets and to test their associations with external variables, a three-step approach was employed (Vermunt 2010) to avoid affecting the composition of latent profiles by covariates. In the first step, a latent profile model was built based on indicator variables, i.e., targets of gratitude. The goal was achieved by employing latent profile analysis (LPA), which is an extension of latent class analysis that uses continuous variables as indicators of profile membership (Collins and Lanza 2010). LPA sorts individuals into groups of subjects who are similar to each other and different from the other groups, based on the individuals' values of indicators. Although this method is similar to traditional clustering procedures (such as K-Means or hierarchical clustering) in terms of its goal, many scholars have pointed out that LPA offers substantial advantages over them: it is more practical and has more power for detecting the optimal number of profiles (Magidson and Vermunt 2002).

Because the expected number of profiles was unknown, an exploratory method of determining the optimal number of profiles was used. Models containing from 1 to 7 profiles were evaluated using the following information criteria based on the model log likelihood: Bayesian Information Criterion (BIC), Akaike Information Criterion (AIC), and the samplesize adjusted BIC (SABIC; Vermunt 2010). Lower values of these indices suggest a better-fitted model. In addition, to assess the precision with which the participants are classified into latent profiles, the entropy value was calculated. A higher value of entropy (i.e., closer to 1) indicates better latent profile separation (Berlin et al. 2014). To test the validity of the local independence assumption, bivariate residuals (BVRs) were analyzed. The values of BVRs greater than 3.84 identify correlations between the pairs of indicators that have not been explained by the model at $\alpha=.05$ (Vermunt and Magidson 2005). In addition to these statistical indices, models were also evaluated on their substantive interpretability to determine if the profiles truly represent different categories, rather than being artifacts (Collins and Lanza 2010). For this reason, profile size was also considered when determining the optimal number of profiles: models that produced profiles representing less than $5 \%$ of the total sample were not further examined.

After building a latent profile model, the participants were assigned to latent profiles on the basis of their probability scores (step 2). In the third step, these probability scores were related to external variables (i.e., gender as a predictor and well-being and coping strategies as outcomes). Wald test 
was performed to examine the significance of the relationships between profile membership and all external variables. For gender, profile specific probabilities and the latent profile distribution for different gender values were calculated using ML adjustment method (Vermunt 2010). For well-being and coping strategies, mean differences were tested across the latent profiles, with BCH adjustment method (Bolck et al. 2004). All calculations were performed using Latent GOLD 5.1 (Vermunt and Magidson 2005) and IBM SPSS Statistics version 25 (IBM Corp. 2017). For the second and third steps of the analysis, so-called Step3 in LatentGOLD 5.1 was used to correct for downward-biased estimates of the strength of the relationships resulting from uncertainty in latent profile membership (Vermunt 2010). The datasets generated during and/or analyzed during the current study are available from the author on reasonable request.

\section{Results}

\section{Missing Data and Preliminary Analysis}

The percentage of missing values was $0.79 \%$. The results of Little's test $\left(\chi^{2}(910)=942.4 ; p>.05\right)$ suggested that data were missing completely at random, which made imputation by EM algorithm possible (Allison 2003).

Table 1 presents descriptive statistics and bivariate correlations for all study variables. The participants scored high or moderately high in all the measured targets of gratitude. Young adults were the most grateful to their mothers. Gratitude toward the mother was significantly higher than gratitude toward the father $\left(F=131.91 ; p<.001 ;\right.$ partial $\left.\eta^{2}=.15\right)$, grandparent(s) $\left(F=156.05 ; p<.001 ;\right.$ partial $\left.\eta^{2}=.17\right)$, sibling(s) $(F=257.65$; $p<.001$; partial $\left.\eta^{2}=.25\right)$, the partner $(F=29.48, p<.001$; partial $\left.\eta^{2}=.04\right)$, and friend(s) $(F=220.62 ; p<.001$; partial $\eta^{2}=.22$ ). Moreover, gratitude toward the partner was higher than gratitude toward the father $(F=24.02, p<.001$; partial $\left.\eta^{2}=.03\right)$, grandparent(s) $(F=39.79, p<.001$; partial $\left.\eta^{2}=.05\right)$, sibling(s) $\left(F=58.29, p<.001 ;\right.$ partial $\left.\eta^{2}=.07\right)$, and friend(s) $\left(F=84.35, p<.001\right.$; partial $\left.\eta^{2}=.10\right)$. Additionally, gratitude toward the father was higher than toward friend(s) $\left(F=5.49, p<.001 ;\right.$ partial $\left.\eta^{2}=.02\right)$.

Levels of gratitude toward different people were only weakly or moderately positively related to each other (i.e., all correlation coefficients were lower than .4), which suggested that they measured different constructs and thus justified applying LPA. In addition, gratitude levels correlated weakly with gender, well-being, and coping strategies (Table 1).

\section{LPA Profiles}

Table 2 presents the results of LPA for the levels of gratitude toward different targets. The information criteria BIC, AIC and SABIC decreased consequently with the addition of latent profiles. As Morin et al. (2016) noted, this is frequent with larger samples, and in such a situation, a decision based on "elbow-plot" rather than on the lowest values of information criteria is recommended. In this study, the graphic presentation of the information criteria suggested a five-profile solution. Moreover, the rejection of six- and seven-profile solutions was supported by a very low frequency for the smallest profile, suggesting the instability of such models (Table 2). Based on these results, the six- and seven-profile solutions were rejected, and the four- and five-profile solutions were investigated more closely. All information criteria indicated that the five-profile solution fitted data better than the fourprofile version. Moreover, a conditional bootstrap ("Bootstrap -2LL Diff') procedure showed that the five-profile solution provided a significant improvement over the four-profile solution ( -2 LL Diff $=1590.55 ; p<.001$; Vermunt and Magidson 2005). Similar results supporting the five-profile model were yielded by the analysis of BVRs. For the fourprofile model, five BVRs exceeded the value of 3.84, indicating the violation of the assumption of local independence. By contrast, for the five-profile model, all BVRs turned out to be non-significant. Thus, although the value of entropy was minimally higher for the four-profile than for the five-profile model (.95 vs .94), after taking into consideration all the results, the four-profile solution was ultimately rejected, and the fiveprofile solution was chosen as fitting data best.

Table 3 and Fig. 1 present five latent profiles identified by LPA. The most numerous, Profile 1 (35.4\%), was labeled "primarily grateful to parents." Members of this profile had a high level of gratitude toward both the mother and the father and a medium level of gratitude toward other people. Profile 2 (24.1\%; "primarily grateful to mother and partner") was characterized by high gratitude toward the mother and the partner, somewhat low gratitude toward the father, and medium gratitude toward other people. For Profile 3 (17.1\%; "highly grateful to all measured targets"), levels of gratitude for all measured interpersonal targets were high. Profile 4 (13\%; "primarily grateful to partner and friend(s)") was composed of young adults with high gratitude toward their partner and friend(s) but relatively low gratitude toward immediate family members. The least numerous, Profile 5 (10.4\%; "not highly grateful to any measured target"), grouped people with relatively low levels of gratitude regardless of its target.

\section{Gender as a Covariate of Profile Membership}

The results of Wald test (Wald $=15,39 ; p=.004$ ) showed that gender was a significant predictor of profile membership. The proportion of women to men was higher in individuals primarily grateful to mother and partner (Profile 2) and individuals primarily grateful to partner and friend(s) (Profile 4) than in individuals primarily grateful to parents (Profile $1 ; \mathrm{Wald}_{2-1}=8.03$; 
Table 1 Descriptive statistics and correlations between levels of gratitude toward different interpersonal targets and external variables

\begin{tabular}{|c|c|c|c|c|c|c|c|c|c|}
\hline Variables & M & SD & Range & 1 & 2 & 3 & 4 & 5 & 6 \\
\hline 1. Gratitude toward mother & 4.63 & 0.85 & $1-5$ & 1 & & & & & \\
\hline 2. Gratitude toward father & 4.09 & 1.28 & $1-5$ & $.39 * * *$ & 1 & & & & \\
\hline 3. Gratitude toward grandparent(s) & 4.05 & 1.10 & $1-5$ & $.21 * * *$ & $.28 * * *$ & 1 & & & \\
\hline 4. Gratitude toward sibling(s) & 4.01 & 1.01 & $1-5$ & $.38 * * *$ & $.35 * * *$ & $.34 * * *$ & 1 & & \\
\hline 5. Gratitude toward partner & 4.38 & 0.99 & $1-5$ & $.08 *$ & .01 & $.09^{*}$ & $.18 * * *$ & 1 & \\
\hline 6. Gratitude toward friend(s) & 3.96 & 0.96 & $1-5$ & $.08^{*}$ & $.11 * *$ & $.20 * * *$ & $.23 * * *$ & $.24 * * *$ & 1 \\
\hline 7. Gender & - & - & - & .01 & $.09^{*}$ & $.11^{* *}$ & -.03 & -.05 & -.06 \\
\hline 8. Emotional well-being & 11.95 & 3.14 & $0-15$ & $.08^{*}$ & $.15^{* * *}$ & $.10^{* *}$ & $.14 * * *$ & $.20 * * *$ & $.20 * * *$ \\
\hline 9. Social well-being & 15.86 & 5.34 & $0-25$ & $.11 * *$ & $.20 * * *$ & $.17 * * *$ & $.16^{* * *}$ & $.08^{*}$ & $.28 * * *$ \\
\hline 10. Psychological well-being & 23.31 & 6.39 & $0-30$ & .03 & $.12 * *$ & $.10^{* *}$ & $.08 *$ & $.12 * *$ & $.23 * * *$ \\
\hline 11. Self-distraction & 2.87 & 1.50 & $0-6$ & .05 & -.06 & .01 & .03 & .01 & .01 \\
\hline 12. Active coping & 3.83 & 1.32 & $0-6$ & .03 & .06 & -.05 & .06 & $.14 * * *$ & .04 \\
\hline 13. Denial & 1.39 & 1.49 & $0-6$ & .01 & -.01 & .04 & .04 & $-.10 * *$ & -.06 \\
\hline 14. Substance use & 0.82 & 1.47 & $0-6$ & -.04 & $-.10 * *$ & -.02 & -.07 & $-.14 * * *$ & .03 \\
\hline 15. Emotional support & 3.98 & 1.62 & $0-6$ & $.08 *$ & -.03 & .04 & $.09 *$ & $.22 * * *$ & $.21 * * *$ \\
\hline 16. Instrumental support & 3.74 & 1.55 & $0-6$ & $.10 * *$ & -.01 & $.08 *$ & $.12 * *$ & $.17 * * *$ & $.13^{* * *}$ \\
\hline 17. Behavioral disengagement & 1.46 & 1.40 & $0-6$ & -.03 & -.06 & .03 & -.01 & -.07 & -.06 \\
\hline 18. Venting & 2.88 & 1.56 & $0-6$ & .01 & -.06 & .02 & .04 & .06 & .02 \\
\hline 19. Positive reframing & 3.04 & 1.44 & $0-6$ & -.01 & .04 & .03 & .06 & $.12 * *$ & $.12 * *$ \\
\hline 20. Planning & 3.78 & 1.40 & $0-6$ & .02 & -.01 & .05 & .07 & $.09^{*}$ & .04 \\
\hline 21. Humor & 2.11 & 1.53 & $0-6$ & -.07 & -.03 & .02 & -.01 & -.04 & $.10^{* *}$ \\
\hline 22. Acceptance & 3.19 & 1.42 & $0-6$ & -.01 & -.04 & .03 & .02 & .05 & $.11 * *$ \\
\hline 23. Religion & 1.87 & 2.04 & $0-6$ & $.09 *$ & $.17 * * *$ & $.13 * * *$ & $.08^{*}$ & $.13 * * *$ & $.10 * *$ \\
\hline 24. Self-blame & 2.75 & 1.87 & $0-6$ & .03 & -.03 & -.02 & .03 & -.05 & -.06 \\
\hline
\end{tabular}

M: mean; $S D$ : standard deviation; ${ }^{*} p<.05 ; * * p<.01 ; * * p<.001$. Gender was dummy-coded ( 0 -women, $1-$ men)

$p=.005 ;$ Wald $\left._{4-1}=4.87 ; p=.027\right)$, highly grateful to all measured targets (Profile 3; Wald ${ }_{2-3}=7.95 ; p=.005 ;$ Wald $_{4-3}=$ $5.34 ; p=.021$ ), and not highly grateful to any measured target (Profile 5; Wald $_{2-5}=5.87 ; p=.015 ;$ Wald $_{4-5}=4.32 ; p=.038$ ). Table 4 shows profile-specific probabilities of the covariate and the latent profile distribution for different gender values.

\section{Relationship between Profile Membership and Outcome Variables}

\section{Well-Being}

Table 5 presents mean differences in well-being and coping strategies across the latent profiles. Online Resource 2 shows a summary of the results. All aspects of well-being were affected by profile membership, which was indicated by the significant value of Wald test (Table 5). Young adults who were highly grateful to all measured targets (Profile 3 ) displayed higher emotional, social and psychological well-being than young adults belonging to other profiles. Moreover, people primarily grateful to parents (Profile 1) had higher levels of all aspects of well-being than people primarily grateful to mother and partner (Profile 2), higher social well-being than young adults primarily grateful to partner and friend(s) (Profile 4), and higher emotional and social well-being than people not highly grateful to any measured target (Profile 5). Additionally, people primarily grateful to partner and friend(s) (Profile 4) had higher emotional well-being than people not highly grateful to any measured target (Profile 5).

\section{Coping Strategies}

Significant mean differences in coping strategies across the profiles were noted for 8 out of 14 strategies. Generally speaking, young adults not highly grateful to any measured target (Profile 5) used fewer constructive strategies than people in the other profiles. Specifically, members of this profile used active coping and emotional support less frequently than other people. Those participants also used religion less often compared to people primarily grateful to parents (Profile 1), people highly grateful to all measured targets (Profile 3 ) and people primarily grateful to partner and friend(s) (Profile 4). Moreover, they used less instrumental support compared to people highly grateful to all measured targets (Profile 3) and 
Table 2 Summary of the model selection criteria of LPA

\begin{tabular}{llllllll}
\hline Model & LL & $\# p$ & BIC & AIC & SABIC & Entropy & Smallest profile (\%) \\
\hline 1-Profile & -6618.13 & 12 & $13,315.94$ & $13,260.26$ & $13,277.83$ & - & - \\
2-Profile & -4459.98 & 25 & 9085.96 & 8969.97 & 9006.58 & .93 & 22.6 \\
3-Profile & -2952.61 & 38 & 6157.54 & 5981.23 & 6036.88 & .96 & 21.8 \\
4-Profile & -2708.32 & 51 & 4847.26 & 4610.63 & 4685.31 & .95 & 7.9 \\
5-Profile & $-\mathbf{1 9 1 3 . 0 5}$ & $\mathbf{6 4}$ & $\mathbf{4 2 5 1 . 0 4}$ & $\mathbf{3 9 5 4 . 0 9}$ & $\mathbf{4 0 4 7 . 8 2}$ & $\mathbf{. 9 4}$ & $\mathbf{1 0 . 3}$ \\
6-Profile & -1710.61 & 77 & 3932.50 & 3575.22 & 3687.99 & .96 & 4.1 \\
7-Profile & -1596.96 & 90 & 3791.52 & 3373.93 & 3505.73 & .96 & 2.3 \\
\hline
\end{tabular}

LL: model Log-likelihood; \#p: number of parameters; BIC: Bayesian Information Criterion; AIC: Akaike Information Criterion; SABIC: sample-size adjusted BIC. Bold values represent a best-fitting model

people primarily grateful to mother and partner (Profile 2); and less positive reframing compared to people highly grateful to all measured targets (Profile 3 ) and people primarily grateful to partner and friend(s) (Profile 4). At the same time, however, young adults not highly grateful to any measured target (Profile 5) used some more adaptive strategies: they more often resorted to humor when coping with stress than young adults primarily grateful to parents (Profile 1) and those primarily grateful to mother and partner (Profile 2), and they blamed themselves less than young adults primarily grateful to parents (Profile 1), to mother and partner (Profile 2), and to partner and friend(s) (Profile 4).

A few differences in coping strategies were also noted between young adults in Profiles 1-4. Compared to people primarily grateful to parents (Profile 1), people highly grateful to all measured targets (Profile 3 ) more frequently used emotional and instrumental support, positive reframing, humor and religion; people primarily grateful to partner and friend(s) (Profile 4) more often displayed humor; and people primarily grateful to mother and partner (Profile 2) more frequently used emotional and instrumental support and venting, but they were less frequently engaged in religion. Moreover, young adults primarily grateful to mother and partner (Profile 2) used humor less often than young adults highly grateful to all measured targets (Profile 3) and those primarily grateful to partner and friend(s) (Profile 4); they were also less engaged in positive reframing and religion, and they blamed themselves more often than young adults highly grateful to all measured targets (Profile 3).

\section{Discussion}

As expected, the study revealed the heterogeneity of the studied sample in terms of levels of gratitude toward different interpersonal targets. This finding is important in itself because it shows the need to investigate the contextual characteristics of gratitude, such as its targets, using a personoriented approach. Significantly, the meaningfulness of the established profiles was supported by the relationship between profile membership and external variables like gender, wellbeing, and coping strategies.

Two of the identified profiles corresponded to gratitude understood as a general disposition: members of Profile 3 (17.1\%) consistently had a high level of gratitude toward various people, whereas members of Profile 5 (10.4\%) consistently had a somewhat low level of gratitude regardless of the target. As expected, people highly grateful to all measured

Table 3 Conditional response means, standard deviations, and the range of means of the 5-profile solution

\begin{tabular}{llllllllll}
\hline Profiles & $\%$ & $\mathrm{n}$ & \multicolumn{2}{l}{ Interpersonal targets of gratitude } & Range of means \\
\cline { 3 - 10 } & & & \multicolumn{2}{l}{$\begin{array}{l}\text { Mother } \\
\mathrm{M}(\mathrm{SD})\end{array}$} & $\begin{array}{l}\text { Father } \\
\mathrm{M}(\mathrm{SD})\end{array}$ & $\begin{array}{l}\text { Grandparent(s) } \\
\mathrm{M}(\mathrm{SD})\end{array}$ & $\begin{array}{l}\text { Sibling(s) } \\
\mathrm{M}(\mathrm{SD})\end{array}$ & $\begin{array}{l}\text { Partner } \\
\mathrm{M}(\mathrm{SD})\end{array}$ & $\begin{array}{l}\text { Friend(s) } \\
\text { M (SD) }\end{array}$ \\
\hline Profile 1 & 35.4 & 271 & $5.00(0.02)$ & $5.00(0.04)$ & $4.08(0.89)$ & $4.14(0.76)$ & $4.09(0.96)$ & $3.93(0.80)$ & $3.67-4.83$ \\
Profile 2 & 24.1 & 184 & $5.00(0.03)$ & $2.95(1.01)$ & $3.79(1.05)$ & $3.86(0.88)$ & $4.49(0.79)$ & $3.81(0.85)$ & $3.33-4.50$ \\
Profile 3 & 17.1 & 131 & $5.00(0.03)$ & $5.00(0.05)$ & $5.00(0.04)$ & $5.00(0.04)$ & $5.00(0.04)$ & $4.45(0.73)$ & $4.67-5.00$ \\
Profile 4 & 13.0 & 99 & $3.39(1.04)$ & $3.56(1.20)$ & $3.67(1.18)$ & $3.38(0.90)$ & $5.00(0.04)$ & $4.14(0.82)$ & $3.00-4.33$ \\
Profile 5 & 10.4 & 80 & $3.41(0.97)$ & $2.84(1.07)$ & $3.50(0.98)$ & $3.10(0.87)$ & $3.27(0.98)$ & $3.40(0.88)$ & $2.33-3.83$ \\
\hline
\end{tabular}

$\mathrm{M}=$ mean; $\mathrm{SD}=$ standard deviation. Theoretical points for gratitude items ranged from 1 to 5 . Profile 1: primarily grateful to parents; Profile 2: primarily grateful to mother and partner; Profile 3: highly grateful to all measured targets; Profile 4: primarily grateful to partner and friend(s); Profile 5: not highly grateful to any measured target 
Fig. 1 Five-profile model of aspects of gratitude

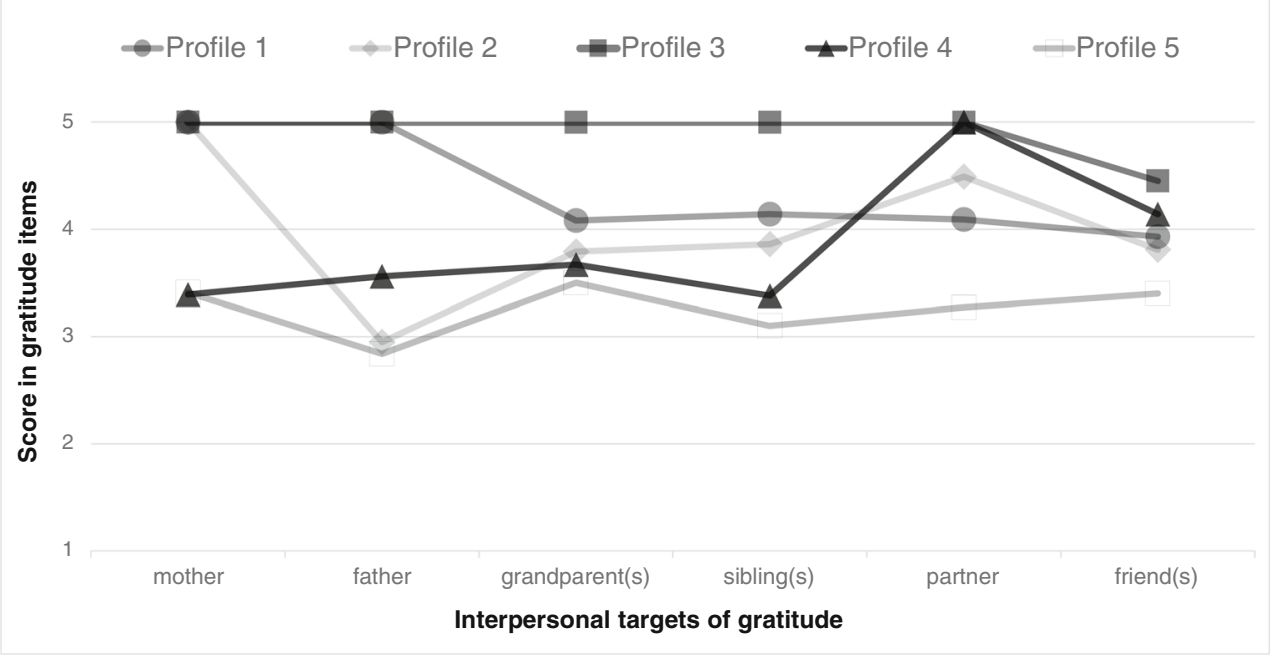

targets displayed higher well-being and generally used more constructive coping strategies than members of the other profiles. By contrast, people with relatively low gratitude toward all measured targets had a lower level of well-being than most profiles (except for Profile 2: primarily grateful to mother and partner) and less often used active coping and emotional support than members of other profiles; compared to most profiles (again, except for Profile 2), they also used religion less often. These results are consistent with the results of studies showing positive relationships between gratitude and well-being (Nezlek et al. 2019; Wood et al. 2010), adaptive coping (Lau and Cheng 2017; Wood et al. 2007) and religiosity (Emmons and Kneezel 2005; Rosmarin et al. 2011).

These two profiles accounted for $27.5 \%$ of the whole sample. The other young adults manifested varied levels of gratitude depending on the target. People who were most diversified in terms of the levels of gratitude toward different targets were grouped in Profiles 2 and 4. Members of Profile 2 felt a high level of gratitude toward their mother and partner, but a somewhat low level of gratitude toward the father, and a medium level of gratitude toward the grandparent(s), sibling(s) and

Table 4 The results of step-three analysis for gender as a predictor of profile membership

\begin{tabular}{|c|c|c|c|c|c|}
\hline & Profile 1 & Profile 2 & Profile 3 & Profile 4 & Profile 5 \\
\hline \multicolumn{6}{|c|}{ Profile specific probabilities of gender } \\
\hline Female & .595 & .729 & .573 & .726 & .571 \\
\hline Male & .405 & .271 & .427 & .274 & .429 \\
\hline \multicolumn{6}{|c|}{ Latent profile distribution for genders } \\
\hline Female & .330 & .275 & .154 & .149 & .093 \\
\hline Male & .396 & .180 & .202 & .099 & .121 \\
\hline
\end{tabular}

Profile 1: primarily grateful to parents (35.4\%); Profile 2: primarily grateful to mother and partner $(24.1 \%)$; Profile 3 : highly grateful to all measured targets (17.1\%); Profile 4: primarily grateful to partner and friend(s) (13\%); Profile 5: not highly grateful to any measured target (10.4\%) friend(s). Individuals from Profile 4, who were primarily grateful to partner and friend(s), seemed to compensate for their potentially unsatisfactory relationships with family members by reorienting their gratitude toward people from outside the family of origin: in this case, the partner and friend(s). Interestingly, the proportion of women to men was higher in those two profiles than in other profiles. This result suggests more complicated associations between gender and latent profile membership than were hypothesized on the basis of the results of previous studies. This leads to the initial hypothesis that women may have a tendency toward greater differentiation of gratitude depending on the target, which in the case of weakened relationships with immediate family member(s) translates to a focus of gratitude on close people outside the family. It is also worth noticing that people belonging to those two profiles have a relatively low level of well-being compared with people highly grateful to all measured targets (Profile 3 ) and, to a lesser degree, people primarily grateful to parents (Profile 1). This result suggests that relatively low gratitude toward the father (Profile 2) or family members in general (Profile 4) may have a negative impact on one's level of well-being despite having a high level of gratitude to non-family close people.

The results of comparing of well-being and coping strategies between Profiles 4 and 5 seem intriguing. What is the difference between people with relatively low levels of gratitude toward most family members but high gratitude toward the partner and friend(s) and people with somewhat low levels of gratitude regardless of the target? First, the former group is more satisfied with their lives and experiences more positive emotions than the latter one. Second, regarding coping strategies, people primarily grateful to non-family close people more often seek emotional support and more often use active coping, positive reframing and religion than people with relatively low gratitude toward all measured targets. On the one hand, they can engage their cognitive resources better than people from Profile 5 to transform and confront the problem, and on the other hand, they 
are open to seeking emotional support and relief both in horizontal relationships (with partner and friend(s)) and vertical ones (with God/Higher Being, etc.). Thus, although they may pay some costs in terms of well-being due to the relatively low level of gratitude to most family members, their high level of gratitude to the partner and friend(s) seems to be a protecting factor, providing them with relatively adaptive coping strategies (Lau and Cheng 2017; Lin 2015).

In comparing these two profiles, it should also be pointed out that people not highly grateful to any measured target less often felt self-blame in stressful situations compared to people primarily grateful to partner and friend(s) (as well as compared to people primarily grateful to parents (Profile 1) and to mother and partner (Profile 2)). On this basis, it can be supposed that young adults with relatively low gratitude regardless of the target make external attributions of their failures, which may lead to grudges and strong feelings of resentment toward others. These processes may make forgiveness difficult for them, which also blocks the arousal of gratitude and prevents them from changing their perspective to be able to appreciate what they receive from others (Luthans et al. 2015).

Having described differences between people primarily grateful to partner and friends and people not highly grateful to any measured target, it is worth pointing out one similarity between people from these profiles. They used humor as a strategy of coping with stress more often than people primarily grateful to parents and people primarily grateful to mother and partner (Profiles 1 and 2). Perhaps these individuals, presumably not having strong bonds with the members of their families, are especially inclined to resort to humor for affiliative and self-enhancement purposes (Martin et al. 2003). However, it should be mentioned that in the Brief COPE (Carver 1997), humor is measured using two quite general items: "I've been making jokes about it" and "I've been making fun of the situation." Since previous studies using this tool have not shown clearly whether the use of such operationalized humor is a constructive coping strategy (see Litman 2006), in future studies, it would be worth applying specific instruments measuring styles of humor, considering both their adaptive and maladaptive functions (e.g., Humor Styles Questionnaire; Martin et al. 2003).

\section{Strengths and Limitations of the Study}

To the best of the author's knowledge, this is the first study to explore the relationships between levels of trait gratitude

Table 5 Relationship between LPA membership and outcome variables

\begin{tabular}{|c|c|c|c|c|c|c|c|c|c|c|c|c|c|c|c|c|}
\hline \multirow[t]{2}{*}{ Outcomes } & \multirow[t]{2}{*}{ Wald } & \multicolumn{5}{|c|}{ Estimated means for LPA profiles } & \multicolumn{10}{|c|}{ Paired comparisons between profiles } \\
\hline & & M1 & M2 & M3 & M4 & M5 & $\begin{array}{l}1 \\
2\end{array}$ & $\begin{array}{l}1 \\
3\end{array}$ & $\begin{array}{l}1 \\
4\end{array}$ & $\begin{array}{l}1 \\
5\end{array}$ & $\begin{array}{l}2 \\
3\end{array}$ & $\begin{array}{l}2 \\
4\end{array}$ & $\begin{array}{l}2 \\
5\end{array}$ & $\begin{array}{l}3 \\
4\end{array}$ & $\begin{array}{l}3 \\
5\end{array}$ & $\begin{array}{l}4 \\
5\end{array}$ \\
\hline \multicolumn{17}{|l|}{ Well-being } \\
\hline Emotional & $47.90 * * *$ & 12.12 & 11.26 & 13.25 & 12.06 & 10.67 & 8.40 & 13.82 & & 11.69 & 33.86 & & & 7.42 & 31.76 & 7.03 \\
\hline Social & $52.28 * * *$ & 16.41 & 14.47 & 18.23 & 15.01 & 14.39 & 14.25 & 10.51 & 5.25 & 9.72 & 38.08 & & & 21.52 & 27.87 & \\
\hline Psychological & $26.84 * * *$ & 23.55 & 21.81 & 25.38 & 23.44 & 22.34 & 8.19 & 7.40 & & & 24.24 & & & 4.49 & 12.34 & \\
\hline \multicolumn{17}{|l|}{ Coping strategies } \\
\hline Self-distraction & 7.12 & 2.72 & 3.06 & 3.02 & 2.73 & 2.92 & & & & & & & & & & \\
\hline Active coping & $11.18^{*}$ & 3.83 & 3.79 & 3.95 & 4.05 & 3.43 & & & & 6.02 & & & 4.41 & & 8.09 & 9.01 \\
\hline Denial & 1.22 & 1.35 & 1.48 & 1.39 & 1.30 & 1.45 & & & & & & & & & & \\
\hline Substance use & 8.56 & 0.72 & 0.86 & 0.84 & 0.65 & 1.24 & & & & & & & & & & \\
\hline Emotional support & $23.42 * * *$ & 3.82 & 4.25 & 4.22 & 4.12 & 3.32 & 7.85 & 5.14 & & 5.72 & & & 17.15 & & 14.16 & 9.94 \\
\hline Instrumental support & $20.93 * * *$ & 3.60 & 4.03 & 3.98 & 3.70 & 3.24 & 9.08 & 5.23 & & & & & 15.39 & & 11.51 & \\
\hline Behavioral disengagement & 5.04 & 1.40 & 1.44 & 1.37 & 1.52 & 1.80 & & & & & & & & & & \\
\hline Venting & $11.48^{*}$ & 2.66 & 3.14 & 2.95 & 2.96 & 2.77 & 10.25 & & & & & & & & & \\
\hline Positive reframing & $16.93^{* *}$ & 2.92 & 2.95 & 3.44 & 3.24 & 2.79 & & 11.31 & & & 8.48 & & & & 10.73 & 4.64 \\
\hline Planning & 6.14 & 3.74 & 3.86 & 3.86 & 3.91 & 3.47 & & & & & & & & & & \\
\hline Humor & $13.52 * *$ & 1.98 & 1.89 & 2.34 & 2.37 & 2.39 & & 4.11 & 4.81 & 4.91 & 5.57 & 6.02 & 6.31 & & & \\
\hline Acceptance & 5.76 & 3.07 & 3.18 & 3.36 & 3.37 & 3.11 & & & & & & & & & & \\
\hline Religion & $24.10^{* * *}$ & 1.99 & 1.48 & 2.44 & 1.92 & 1.31 & 6.88 & 3.94 & & 8.23 & 15.61 & & & & 16.43 & 4.18 \\
\hline Self-blame & $10.68 *$ & 2.78 & 2.94 & 2.49 & 2.98 & 2.31 & & & & 3.98 & 4.62 & & 6.47 & & & 5.40 \\
\hline
\end{tabular}

${ }^{*} p<.05 ; * *<.01 ; * * * p<.001 ; M 1-M 5$ : estimated means for profiles $1-5$. Profile 1 : primarily grateful to parents (35.4\%); Profile 2: primarily grateful to mother and partner (24.1\%); Profile 3: highly grateful to all measured targets (17.1\%); Profile 4: primarily grateful to partner and friend(s) (13\%); Profile 5: not highly grateful to any measured target $(10.4 \%)$. For mean differences between the latent profiles, only Wald's values significant at least at .05 are presented 
toward different interpersonal targets using the personoriented approach. Numerous differences in gender, wellbeing and coping strategies across the latent profiles support the meaningfulness of the established profiles and concurrent validity of LPA-generated model (Berlin et al. 2014).

Despite a number of strengths, this study has some limitations that should be addressed. First, although the test-retest coefficients for gratitude items were satisfactory in the current study, using single items to assess the levels of gratitude could reduce intra- and inter-individual variability. Nonetheless, gratitude items turned out to be sufficiently sensitive to capture differences in levels of gratitude toward different people across the profiles. Second, the study did not consider the characteristics of the family of origin (such as having divorced or separated parents or growing up in a single-parent family) as a potential covariate of profile membership. Third, only some of the most likely targets of gratitude, being members of immediate family and non-family close people, were included in the study. It cannot be discounted that the young adults who were not highly grateful to any of the six targets considered in this study may have been highly grateful to other people. Thus, in future research it is recommended to take into account other interpersonal targets such as uncles and aunts, teachers, neighbors, doctors, psychologists, and acquaintances.

Another limitation of the study stems from the sample, which was collected using convenience sampling. Further research is needed to examine whether the number and shapes of the profiles will be replicated across other samples.

When interpreting relationships between latent profile membership and outcome variables, the cross-sectional design of the study should be taken into account. Because the directions of these relationships were solely based on theoretical considerations and evidence from previous studies, causal relationships between the variables cannot be inferred. However, the primary purpose of this study was to demonstrate the usefulness of a person-oriented approach in examining gratitude by providing evidence for the heterogeneity of the sample with respect to levels of trait gratitude toward different people. After examining the similarities of profile solutions in other samples, it would be worthwhile in future studies to take a closer look at causal relationships and mechanisms involved in both the antecedents and consequences of these profiles.

\section{Conclusions}

This study proved that the sample of young adults was heterogeneous with regard to the levels of gratitude toward different interpersonal targets. Only approximately $28 \%$ of young adults had a similar level of gratitude irrespective of the target. In the other individuals, differences were found in terms of the level of gratitude depending on its target. What is important is that belonging to different profiles of gratitude was determined by gender and was related to important outcomes such as wellbeing and coping strategies. This study suggests that, at least in the case of some research problems, both considering differences in trait gratitude depending on the target and using a person-oriented approach to explore that gratitude would provide a more holistic picture for the complex nature of gratitude and its relationships with other variables. In addition, studying this issue using the person-oriented approach may provide practical guidelines for consultants and therapists in their work with clients, tailored to the specific needs of subgroups of clients who struggle with the feeling of being ungrateful toward a particular member of the family, partner or friend.

\section{Compliance with Ethical Standards}

Conflict of Interest The author states that there is no conflict of interest.

Ethical Approval All procedures performed in studies involving human participants were in accordance with the ethical standards of the Research Ethics Committee at the Faculty of Pedagogy and Psychology of the University of Silesia in Katowice, Poland, and with the 1964 Helsinki declaration and its later amendments or comparable ethical standards.

The protocol received a written approval in Polish from the Research Ethics Committee at the Faculty of Pedagogy and Psychology of the University of Silesia in Katowice, Poland. It states that the Research Ethics Committee considered the ethical aspects of the submitted research project on the basis of the provided documentation:

1. Application to the Research Ethics Committee at the Faculty of Pedagogy and Psychology of the University of Silesia in Katowice to receive their opinion on the research project.

2. Attachment no. 1: Additional information on the research project.

3. Attachment no. 2: The researcher's declaration - obligation to obtain an informed written consent from an adult.

Based on these documents, the Research Ethics Committee unanimously decided that the submitted project did not raise any ethical concerns.

Informed Consent Informed consent was obtained from all individual participants included in the study. It consisted of the following elements: the purpose of the study, a statement regarding confidentiality and anonymity of participants, a statement regarding the participant's right to withdraw her or his consent at any time and the full name and contact details of PI.

Open Access This article is distributed under the terms of the Creative Commons Attribution 4.0 International License (http:// creativecommons.org/licenses/by/4.0/), which permits unrestricted use, distribution, and reproduction in any medium, provided you give appropriate credit to the original author(s) and the source, provide a link to the Creative Commons license, and indicate if changes were made.

\section{References}

Algoe, S. B., Haidt, J., \& Gable, S. L. (2008). Beyond reciprocity: Gratitude and relationships in everyday life. Emotion, 8(3), 425-429.

Allison, P. D. (2003). Missing data techniques for structural equation models. Journal of Abnormal Psychology, 112(4), 545-557.

Bergman, L. R., Magnusson, D., \& El-Khouri, B. M. (2003). Studying individual development in an interindividual context: A personoriented approach. Mahwah: Erlbaum. 
Berlin, K. S., Williams, N. A., \& Parra, G. R. (2014). An introduction to latent variable mixture modeling (part 1): Overview and crosssectional latent class and latent profile analyses. Journal of Pediatric Psychology, 39(2), 174-187.

Bolck, A., Croon, M. A., \& Hagenaars, J. A. (2004). Estimating latent structure models with categorical variables: One-step versus threestep estimators. Political Analysis, 12(1), 3-27.

Carver, C. S. (1997). You want to measure coping but your protocol is too long: Consider the brief Cope. International Journal of Behavioral Medicine, 4(1), 92-100.

Carver, C. S., Scheier, M. F., \& Weintraub, J. K. (1989). Assessing coping strategies: A theoretically based approach. Journal of Personality and Social Psychology, 56(2), 267-283.

Chen, L. H. (2017). Gratitude and athletes' well-being. In A. Brady \& B. Grenville-Cleave (Eds.), Positive psychology in sport and physical activity: An introduction (pp. 129-139). New York: Routledge/ Taylor \& Francis Group.

Chen, L. H., \& Chang, Y.-P. (2017). Sport-domain gratitude uniquely accounts for athletes' well-being across two cultures: Incremental validity above the general gratitude. The Journal of Positive Psychology, 12(6), 651-659.

Collins, L. M., \& Lanza, S. T. (2010). Latent class and latent transition analysis: With applications in the social, behavioral, and health sciences. Hoboken: John Wiley and Sons Inc..

Emmons, R. A., \& Kneezel, T. T. (2005). Giving thanks: Spiritual and religious correlates of gratitude. Journal of Psychology and Christianity, 24(2), 140-148.

Emmons, R. A., \& McCullough, M. E. (2003). Counting blessings versus burdens: An experimental investigation of gratitude and subjective well-being in daily life. Journal of Personality and Social Psychology, 84(2), 377-389.

Enright, R., \& The Human Development Study Group. (1991). The moral development of forgiveness. In W. Kurtines \& J. Gewirtz (Eds.), Handbook of moral behavior and development (pp. 123-152). Hillsdale, NJ: Erlbaum.

Fan, W., \& Yan, Z. (2010). Factors affecting response rates of the web survey: A systematic review. Computers in Human Behavior, 26(2), 132-139.

Froh, J. J., Bono, G., \& Emmons, R. (2010). Being grateful is beyond good manners: Gratitude and motivation to contribute to society among early adolescents. Motivation and Emotion, 34(2), 144-157.

Galesic, M., \& Bosnjak, M. (2009). Effects of questionnaire length on participation and indicators of response quality in a web survey. Public Opinion Quarterly, 73(2), 349-360.

Glover, R. J. (2000). Developmental tasks of adulthood: Implications for counseling community college students. Community College Journal of Research \& Practice, 24(6), 505-514.

Gordon, C. L., Arnette, R., \& Smith, R. E. (2011). Have you thanked your spouse today?: Felt and expressed gratitude among married couples. Personality and Individual Differences, 50(3), 339-343.

Graham, S. (1988). Children's developing understanding of the motivational role of affect: An attributional analysis. Cognitive Development, 3(1), 71-88.

Hammer, J. H., \& Brenner, R. E. (2019). Disentangling gratitude: A theoretical and psychometric examination of the gratitude resentment and appreciation test- revised short (GRAT-RS). Journal of Personality Assessment, 101(1), 96-105.

Hall, J. H., \& Fincham, F. D. (2005). Self-forgiveness: The stepchild of forgiveness research. Journal of Social and Clinical Psychology, 24(5), 621-637.

Hill, P. L., Allemand, M., \& Roberts, B. W. (2013). Examining the pathways between gratitude and self-rated physical health across adulthood. Personality and Individual Differences, 54(1), 92-96.

IBM Corp. Released. (2017). IBM SPSS statistics for windows, version 25.0. Armonk: IBM Corp.
Jans-Beken, L. G., Jacobs, N., Janssens, M., Peeters, S., Reijnders, J., Lechner, L., \& Lataster, J. (2018). Reciprocal relationships between state gratitude and high- and low-arousal positive affects in daily life: A time-lagged ecological assessment study. The Journal of Positive Psychology, 14(4), 512-527.

Juczyński, N., \& Ogińska-Bulik. (2009). Narzedzia pomiaru stresu $i$ radzenia sobie ze stresem [instruments to measure stress and coping with stress]. Warszawa: Pracownia Testów Psychologicznych.

Karaś, D., Cieciuch, J., \& Keyes, C. L. M. (2014). The polish adaptation of the mental health continuum-short form (MHC-SF). Personality and Individual Differences, 69, 104-109.

Kashdan, T. B., Mishra, A., Breen, W. E., \& Froh, J. J. (2009). Gender differences in gratitude: Examining appraisals, narratives, the willingness to express emotions, and changes in psychological needs. Journal of Personality, 77(3), 691-730.

Keyes, C. L. M. (2002). The mental health continuum: From languishing to flourishing in life. Journal of Health and Social Behavior, 43(2), 207-222.

Krause, N., Emmons, R. A., Ironson, G., \& Hill, P. C. (2017). General feelings of gratitude, gratitude to god, and hemoglobin A1c: Exploring variations by gender. The Journal of Positive Psychology, 12(6), 639-650.

Lau, B. H., \& Cheng, C. (2017). Gratitude and coping among familial caregivers of persons with dementia. Aging \& Mental Health, 21(4), 445-453.

Layous, K., Sweeny, K., Armenta, C., Na, S., Choi, I., \& Lyubomirsky, S. (2017). The proximal experience of gratitude. PLoS One, 12(7), e0179123.

Lenz, B. (2001). The transition from adolescence to young adulthood: A theoretical perspective. The Journal of School Nursing, 17(6), 300-306.

Lin, C. C. (2015). Impact of gratitude on resource development and emotional well-being. Social Behavior and Personality: An International Journal, 43, 493-504.

Lin, C. C. (2017). The effect of higher-order gratitude on mental wellbeing: Beyond personality and unifactorial gratitude. Current Psychology, 36(1), 127-135.

Litman, J. A. (2006). The COPE inventory: Dimensionality and relationships with approach- and avoidance-motives and positive and negative traits. Personality and Individual Differences, 41(2), 273-284.

Little, R. J., \& Rubin, D. B. (1987). Statistical analysis with missing data. New York, NY: John Wiley \& Sons.

Luthans, F., Youssef-Morgan, C. M., \& Avolio, B. J. (2015). Psychological capital and beyond. Oxford: Oxford University Press.

Ma, L. K., Tunney, R. J., \& Ferguson, E. (2017). Does gratitude enhance prosociality?: A meta-analytic review. Psychological Bulletin, 143(6), 601-635.

Macaskill, A. (2012). Differentiating dispositional self-forgiveness from other-forgiveness: Associations with mental health and life satisfaction. Journal of Social and Clinical Psychology, 31(1), 28-50.

Magidson, J., \& Vermunt, J. K. (2002). Latent class models for clustering: A comparison with K-means. Canadian Journal of Marketing Research, 20, 36-43.

Martin, R. A., Puhlik-Doris, P., Lersen, G., Gray, J., \& Weir, K. (2003). Individual differences in uses of humor and their relation to psychological wellbeing: Development of the humor styles questionnaire. Journal of Research in Personality, 37(1), 48-75.

McCullough, M. E., Emmons, R. A., \& Tsang, J. (2002). The grateful disposition: A conceptual and empirical topography. Journal of Personality and Social Psychology, 82(1), 112-127.

Meade, A. W., \& Craig, S. B. (2012). Identifying careless responses in survey data. Psychological Methods, 17(3), 437-455.

Morgan, B., Gulliford, L., \& Kristjánsson, K. (2017). A new approach to measuring moral virtues: The multi-component gratitude measure. Personality and Individual Differences, 107, 179-189. 
Morin, A., Meyer, J. P., Creusier, J., \& Bietry, F. (2016). Multiple-group analysis of similarity in latent profile solutions. Organizational Research Methods, 19(2), 231-254.

Nezlek, J. B., Krejtz, I., Rusanowska, M., \& Holas, P. (2019). Withinperson relationships among daily gratitude, well-being, stress, and positive experiences. Journal of Happiness Studies, 20(3), 883-898.

Peterson, C., \& Seligman, M. (2004). Character strengths and virtues: A handbook and classification. Washington, DC: APA Press.

Rolstad, S., Adler, J., \& Rydén, A. (2011). Response burden and questionnaire length: Is shorter better? A review and meta-analysis. Value in Health, 14(8), 1101-1108.

Rosmarin, D. H., Pirutinsky, S., Cohen, A. B., Galler, Y., \& Krumrei, E. J. (2011). Grateful to god or just plain grateful? A comparison of religious and general gratitude. The Journal of Positive Psychology, 6(5), 389-396.

Ross, S. R., Kendall, A. C., Matters, K. G., Wrobel, T. A., \& Rye, M. S. (2004). A personological examination of self-and other-forgiveness in the five-factor model. Journal of Personality Assessment, 82(2), 207-214.

Rossiter, J. R. (2002). The C-OAR-SE procedure for scale development in marketing. International Journal of Research in Marketing, 19(4), 305-335.

Sackett, P. R., \& Larson, J. R. (1990). Research strategies and tactics in industrial and organizational psychology. In M. D. Dunnette \& L. M. Hough (Eds.), Handbook of industrial and organizational psychology (pp. 419-489). Palo Alto: Consulting Psychologists Press.

Svalina, S. S., \& Webb, J. R. (2012). Forgiveness and health among people in outpatient physical therapy. Disability and Rehabilitation, 34(5), 383-392.

Toepoel, V., Das, M., \& van Soest, A. (2009). Design of web questionnaires: The effects of the number of items per screen. Field Methods, 21(2), 200-213.

Toussaint, L., \& Webb, J. (2005). Theoretical and empirical connections between forgiveness, mental health, and well-being. In W. E. L.
Worthington (Ed.), Handbook of forgiveness (pp. 349-362). New York: Brunner-Routledge.

Toussaint, L. L., Williams, D. R., Musick, M. A., \& Everson, S. A. (2001). Forgiveness and health: Age difference in a U.S. probability sample. Journal of Adult Development, 8(4), 249-257.

Vermunt, J. K. (2010). Latent class modeling with covariates: Two improved three-step approaches. Political Analysis, 18(4), 450-469.

Vermunt, J. K., \& Magidson, J. (2005). Latent GOLD 4.0 User's Guide. Belmont: Statistical Innovations Inc.

Visserman, M. L., Righetti, F., Impett, E. A., Keltner, D., \& Van Lange, P. A. M. (2018). It's the motive that counts: Perceived sacrifice motives and gratitude in romantic relationships. Emotion, 18(5), 625-637.

Watkins, P., Woodward, K., Stone, T., \& Kolts, R. (2003). Gratitude and happiness: Development of a measure of gratitude and relationships with subjective well-being. Social Behavior and Personality: An International Journal, 31, 431-452.

Webb, J. R., Robinson, E. A. R., Brower, K. J., \& Zucker, R. A. (2006). Forgiveness and alcohol problems among people entering substance abuse treatment. Journal of Addictive Diseases, 25(3), 55-67.

Wood, A. M., Joseph, S., \& Linley, P. A. (2007). Coping style as a psychological resource of grateful people. Journal of Social and Clinical Psychology, 26(9), 1076-1093.

Wood, A. M., Maltby, J., Gillett, R., Linley, P. A., \& Joseph, S. (2008). The role of gratitude in the development of social support, stress, and depression: Two longitudinal studies. Journal of Research in Personality, 42(4), 854-871.

Wood, A. M., Froh, J. J., \& Geraghty, A. W. (2010). Gratitude and wellbeing: A review and theoretical integration. Clinical Psychology Review, 30(7), 890-905.

Publisher's note Springer Nature remains neutral with regard to jurisdictional claims in published maps and institutional affiliations. 